\title{
Time-Course Sensitive Collision Probability Model for Risk Estimation
}

\author{
Fabian Müller $^{1}$ and Julian Eggert ${ }^{2}$
}

\begin{abstract}
Avoiding critical situations is a prerequisite for Advanced Driver Assistant Systems and Autonomous Driving to decrease the number of total hazards and fatal collisions. As a guide for safe motion behavior and for avoiding critical situations in complex scenarios with several interacting traffic participants, an appropriate risk measurement is necessary. It should incorporate system-inherent uncertainties like present in environment recognition, behavior predictions and physical model assumptions. In this paper, we introduce a time-courseaware incremental risk model for motion planning which predicts state distributions along forecasted trajectories and regards their magnitude evolution by the Survival Theory and their shape adaptation by removing collided distribution parts while preserving statistical moments. Our approach is able to reproduce motion risk probability costs as found by particlebased Monte-Carlo (MC) simulations in a range of scenarios, at much lower computational costs.
\end{abstract}

\section{INTRODUCTION}

The ambitious zero-accident target of institutions and car makers is a driving force for continued research in the area of risk estimation for intelligent Advanced Driver Assistant Systems (ADAS) and Autonomous Driving (AD). To approach that target and to obtain acceptance of driver-less vehicles, such systems have to deal with all kinds of complex traffic scenarios and find those paths and maneuvers which are less likely to end up in crashes. Corresponding motion planners should consider all kinds of risks like crashes or loss of control caused by street infrastructure and car dynamics. This can be done by modeling these different risk types with a holistic risk approach, so that no unreasonable behavior occurs.

With this purpose in mind, the avoidance of collisions between two participants is a challenging problem. Imperfect sensors and measurements as well as uncertain motion behavior of other traffic participants like vehicles or pedestrians prohibit the calculation of a single deterministic future evolution of the scene. To handle an uncertain future, a holistic risk concept has to regard several future outcomes together with their probability and their severity [1]. In many applications [2]-[5], the formulation of a risk which consists of a probability model for critical events and mostly a simple constant severity model [6], works as a safety measure and helps controllers or human planner to avoid or mitigate unsafe future scenarios.

${ }^{1}$ Fabian Müller is with the Control Methods and Robotics Lab, Technical University of Darmstadt, 64283 Darmstadt, Germany fabian.muel lerermr.tu-darmstadt.de

${ }^{2}$ Julian Eggert is with the Honda Research Institute (HRI) Europe, Carl-Legien-Str. 30, 63073 Offenbach/Main, Germany julian.eggertahonda-ri.de
For capturing uncertainties and their future evolution during prediction, in addition to sampling approaches Kalman filters [7] (e.g. for tracking positions, velocities or orientations) and multidimensional Gaussian state distribution [2] are commonly used. Transition models for all relevant traffic participants provide future state distributions, and collision probabilities can be gained by evaluation of the distribution w.r.t. e.g. the geometric overlap of the shapes of the involved entities, given their predicted states. Depending on the details of the form and overlap model assumptions, different methods are available to calculate the overlap probability of circularly shaped [4], [8], non-convex polygonal-shaped [2] and point approximated entities for far distant scenarios [9]. For more complex shaped entity overlap probability calculation Monte Carlo (MC) simulations are also used, see e.g. [10].

From the time-course of future overlap probabilities or predicted risks, a single integral risk value representative for the current time $t$ needs to be calculated. For this purpose, in [8], [11] and [12], this integral risk is extracted from the maximum of the predicted risks, neglecting all other contributions which get invisible to a planner. Other approaches take the integral of the risk along the trajectory over time. In [4], the authors use a flow calculation technique to estimate the cumulative risk of entering a circular safety zone around two ships. The direction of the probability density flow through the boundaries of a collision region is considered in [13]. Unfortunately, these approaches are usually not sensitive to the prediction time-course of the predicted risk. To address this, the Survival Theory, described in [14], provides a probabilistic model for the time-course-sensitive probability reduction along the prediction time. This was used in [15], [16] for the construction of a simple integral risk-based motion planner capable of handling a range of parallel lane scenarios with multiple vehicles based on incremental utility, risk and comfort terms. Other works incorporate the prediction time-course by the incremental extraction of already collided parts of a distribution. [17] represents future states by enlarging circles where collided areas are removed for the collision probability calculation. In another approach, a bunch of possible trajectories is propagated into the future by an exhaustive MC simulation, and the collision probability is calculated by the proportion of collided trajectories [6]. In [18], the Gaussian state distributions are propagated and truncated by linear boundary constraints arising from fixed obstacles. In this paper, we present a time-course sensitive many-entity collision probability model for based on an analytic approximation of the distribution along predicted trajectories to calculate time-incremental risks. For this, we 
connect the survival theory [14], which predicts the probability of reaching future time points, with an algorithm for the collision sensitive analytic shape adaptation of Gaussian distribution functions for predicted states by given state transitions for all future time points.

\section{Problem Statement}

We consider a driving scene at time $t$, involving a vehicle $i$ that interacts with other traffic participants $j$. The task is to estimate the collision risk, and with it, the collision probability, of $i$ with any other vehicle for some future time period $\left[t, t+s_{\max }\right]$. Since there are different kinds of uncertainties involved in the future development of the scene, we describe the current and future states of all entities by probability distribution functions (pdf's). The uncertainty in the states originates from several sources. On one side, measurement and estimation uncertainties of the current time $t$ initial states like positions, orientations and velocities. On the other side, prediction and modeling errors arise from behavioral uncertainties, the propagation of measurement uncertainties as well as the mismatch with reality of the models that are used to predict the future states of all traffic participants.

The estimation of the collision risk during any intermediate future time $t+s$, with $s \in\left[0, s_{\max }\right]$, will certainly depend on the previous prediction time course during $[t, t+s[$. This is what we call the time-course sensitivity (tcs) of the predicted risk. This should become clear considering e.g. the case that a high collision probability in the distant future $t+s$ can only occur if, during the preceding time $[t, t+s[$, the overall predicted collision risk was low, since otherwise the vehicle $i$ would not have "survived" until $t+s$ in the first place. Or, other way round, if there is a high predicted collision probability at $t+s$, then the collision probability will be low afterwards for $\left.] t+s, t+s_{\max }\right]$, because the probability that the vehicle will have survived the collision at $t+s$ is low.

We now assume that for the prediction of states during $\left[t, t+s_{\max }\right]$ we have a propagation model of the entity pdf's for the collision-free condition. In addition, we assume that at any time step, given the predicted states, we can check whether a collision occurs or not. The question is then how to estimate the history-dependent collision risk function for the predicted time interval.

Inevitably, if we are able to estimate the full sequence of pdf's for the prediction time, which incorporates the influence of the collisions at any time step, then we would have reached our goal. The task therefore breaks down into the estimation of a realistic and efficient model of collisionsensitive future state pdf propagation. This task might be separated again into two subtasks: One is to calculate from pdf's the probability magnitude (i.e., a scalar) that a collision occurs, and propagate this further into the future. The second is to estimate the influence that the collisions might have on the form of the state pdf's, considering in detail which states lead to collisions and which continue to survive for future consideration.

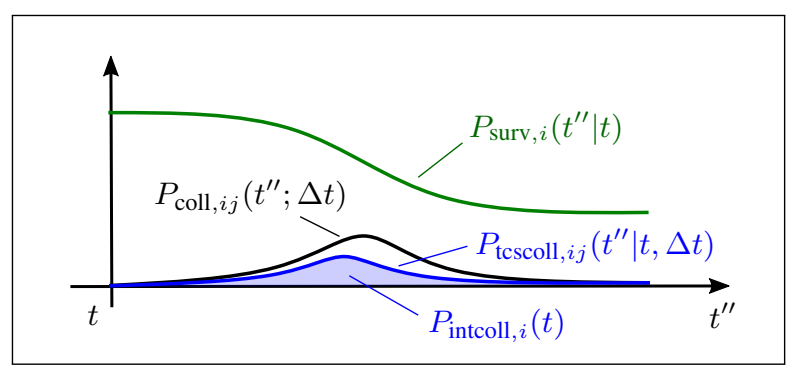

Fig. 1: Survival probability curve $P_{\text {surv }, i}\left(t^{\prime \prime} \mid t\right)$, time-course sensitive prob. curve $P_{\mathrm{tcscoll}, i j}\left(t^{\prime \prime} \mid t, \Delta t\right)$ and integral collision prob. area $P_{\text {intcoll }, i}(t)$ for a given exemplary instantaneous collision event prob. $P_{\text {coll }, i j}\left(t^{\prime \prime} ; \Delta t\right)$ curve as function of predicted time $t^{\prime \prime}$.

The first subtask relates to the problem of estimating the survival probability of vehicle $i$ over $\left[t, t+s_{\max }\right]$. This has been focus of previous work on general risk estimation using survival theory, see e.g. [11]. We will sketch the main idea of the survival theory relevant for this paper in the next section. The second subtask, the time sensitive adaptation of the pdf shape, is the main target of this paper, and will be introduced from section IV-C on. The two subtasks influence each other in the sense that both are necessary for a correct handling of collision risks in specific traffic scenes.

\section{Previous Work: Survival Theory}

Let us denote the predicted probability that the vehicle $i$ survives at a point in time $t^{\prime}>t$, given that we start our prediction at $t$, with the survival probability $P_{\text {surv }, i}\left(t^{\prime} \mid t\right)$, with starting condition $P_{\text {surv }, i}(t \mid t)=1$. What is then the probability $P_{\text {surv }, i}\left(t^{\prime \prime} \mid t\right)$ that vehicle $i$ will still survive at a later moment in time $t^{\prime \prime}$ ? This is in essence what survival theory is about. From probability considerations we easily see that

$$
P_{\text {surv }, i}\left(t^{\prime \prime} \mid t\right)=P_{\text {surv }, i}\left(t^{\prime \prime} \mid t^{\prime}\right) P_{\text {surv }, i}\left(t^{\prime} \mid t\right)
$$

and so on. If we split the interval $\left[t, t^{\prime \prime}\right]$ into ever smaller subintervals with corresponding survival probabilities $P_{\text {surv }, i}$, we see that $P_{\text {surv }, i}\left(t^{\prime \prime} \mid t\right)$ essentially depends on all time steps within $\left[t, t^{\prime \prime}\right]$, i.e., it depends on the entire prediction time-course from $t$ to $t^{\prime \prime}$. Written as $P_{\text {surv }, i}(t+s \mid t)$, we get a survival function that starts at 1 for $s=0$ and is monotonously decreasing for ever increasing $s$, i.e., it can never grow. In addition, for larger (collision) risks, it will decrease faster. So how can we calculate $P_{\text {surv }, i}$ ?

Starting from discrete time intervals of size $\Delta t$, and looking exclusively at collision events for the survival calculation (other risks can be incorporated without loss of generality into these considerations, see e.g. [11] for a general risk theory based on survival probability), we now introduce $P_{\text {coll }, i j}\left(t^{\prime} ; \Delta t\right)$ as the collision probability during the time interval $\left[t^{\prime}, t^{\prime}+\Delta t\right]$. We call this the instantaneous event/ collision probability, since it will depend only on the states around $t^{\prime}$ and not on the time course $\left[t, t^{\prime}\right]$ since prediction start, in the sense that it will be zero if the states of the entities at $t^{\prime}$ are such that no collision takes place. We then have that for the ego-car (car $i$ ), assuming statistical 
independence of the events, the total probability of not colliding with any other entity during the next short time interval $[t, t+\Delta t]$ is given by

$$
P_{\mathrm{nc}, i}(t ; \Delta t)=\prod_{j \neq i}\left[1-P_{\mathrm{coll}, i j}(t ; \Delta t)\right]
$$

and consequently the probability of colliding by

$$
P_{\mathrm{coll}, i}(t ; \Delta t)=1-P_{\mathrm{nc}, i}(t ; \Delta t)
$$

The probability that vehicle $i$ will not be engaged in any of the events in the next time interval, i.e., the probability that the vehicle survives, can be expressed by

$$
\begin{aligned}
P_{\text {surv }, i}(t+\Delta t \mid t) & =P_{\mathrm{nc}, i}(t ; \Delta t) P_{\text {surv }, i}(t \mid t) \\
P_{\text {surv }, i}(t+2 \Delta t \mid t) & =P_{\mathrm{nc}, i}(t+\Delta t ; \Delta t) P_{\text {surv }, i}(t+\Delta t \mid t) \\
P_{\text {surv }, i}(t+n \Delta t \mid t) & =\ldots
\end{aligned}
$$

(starting with $P_{\text {surv }, i}(t \mid t)=1$ ) so that we get

$$
P_{\text {surv }, i}(t+n \Delta t \mid t)=\prod_{k=0}^{n-1} P_{\mathrm{nc}, i}(t+k \Delta t ; \Delta t) P_{\text {surv }, i}(t \mid t) .
$$

For small time intervals $\Delta t \rightarrow 0$ we can set

$$
P_{\mathrm{coll}, i j}(t ; \Delta t) \approx \frac{d}{d t} P_{\mathrm{coll}, i j}(t ; \Delta t) \Delta t:=\tau_{\mathrm{coll}, i j}^{-1}(t) \Delta t
$$

with the collision event rate $\tau_{\text {coll, } i j}^{-1}$ (number of collision events between entity $i$ and $j$ per time unit), which then leads to the total entity $i$ specific event rate

$$
\tau_{\mathrm{coll}, i}^{-1}(t)=\sum_{j \neq i} \tau_{\mathrm{coll}, i j}^{-1}(t)
$$

and, in continuous time, to the survival function

$$
P_{\text {surv }, i}\left(t^{\prime \prime} \mid t\right)=\exp \left\{-\int_{t}^{t^{\prime \prime}} \tau_{\text {coll }, i}^{-1}\left(t^{\prime}\right) d t^{\prime}\right\} \text {. }
$$

These last two equations can be generalized to arbitrary other risks $k$ quantifiable via risk event rates $\tau_{k}^{-1}$, with all risk event contributions adding linearly as in eq. (7).

With the survival function (either calculated discretely eq. (5) or continuously eq. (8)), it becomes easy to calculate the real, time-course sensitive (tcs) predicted risk for each event contribution, in our case e.g. the collision with $j$, according to

$$
P_{\mathrm{tcscoll}, i j}\left(t^{\prime \prime} \mid t, \Delta t\right)=P_{\mathrm{coll}, i j}\left(t^{\prime \prime} ; \Delta t\right) P_{\mathrm{surv}, i}\left(t^{\prime \prime} \mid t\right),
$$

meaning that it is given by the probability that, starting at time $t$, the vehicle $i$ survives potential collisions with all entities until $t^{\prime \prime}$, to then get involved in a collision with entity $j$ during the interval of size $\Delta t$ around $t^{\prime \prime}$. The relationship between the instantaneous probability $P_{\text {coll }, i j}$, the survival probability $P_{\text {surv, } i}$ and the time-course sensitive probability $P_{\text {tcscoll }, i j}$ is exemplarily depicted in Fig. 1. A beneficial side effect is that we can use eq. (9) to calculate the integral probabilities and risk contributions (see e.g. [15]) involved in a planned, predicted ego-vehicle trajectory, simply by adding

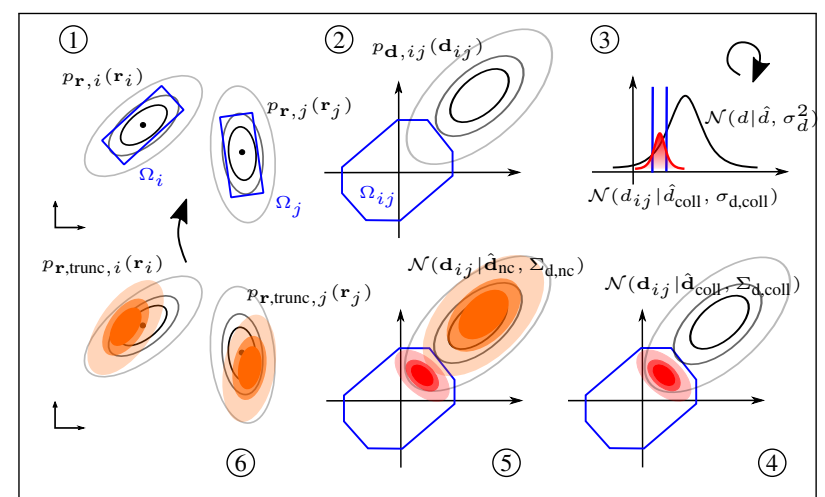

Fig. 2: State probability truncation approach based on Gaussian distributions. See text section IV for details. The approach starts at the upper left corner (point 1), where the current predicted vehicle pdf's and orientation are given. The schema of the truncation process and the back inference on the truncated vehicle state pdf's is indicated by points 1-6.

over all the event contributions (e.g. all other vehicles which might collide) future time,

$$
P_{\text {intcoll }, i}(t):=\sum_{n} \sum_{j} P_{\mathrm{tcscoll}, i j}(t+n \Delta t \mid t, \Delta t),
$$

This is the total probability (for $\Delta t \rightarrow 0$ it can be shown that $\left.P_{\text {intcoll }, i}(t) \in[0,1]\right)$ that vehicle $i$ will get involved in a collision with any other entity at some point in the future.

Summarizing, the survival theory allows to estimate the probability magnitude of certain risks over future time, given a predicted scene time-course. In the next sections, we will determine the instantaneous collision probability value and we will concentrate on the estimation of the form of the future state pdf's. The connection between the two is given by the instantaneous collision probability eq. (3), which is fed from the state pdf's into the survival calculation.

\section{Collision Probability Calculation ApProACH}

\section{A. 2-Entity Instantaneous Overlap Probability}

We assume collision entities (in our case, vehicles) with geometries which can be described by 2D shape polygons $\Omega_{j}\left(\mathbf{r}_{j}, \theta_{j}\right)$ anchored at specific 2D positions $\mathbf{r}_{j}$ and with orientations $\theta_{j}$. We consider a collision of 2 entities to occur when their respective polygons touch or overlap.

The positions and orientations can be uncertain so that the overall location of a vehicle would be described by a probability distribution function (pdf) over 2D position and orientation. In this paper, and since it is the more relevant parameter (the vehicle orientation is often tangential to the vehicle trajectory), we consider the orientations to be deterministic and only the positions to be uncertain, with the positions described by Gaussian pdf's,

$\mathcal{N}(\mathbf{r} \mid \hat{\mathbf{r}}, \Sigma):=\frac{1}{2 \pi \sqrt{\operatorname{det}\left[\Sigma_{\mathbf{r}}\right]}} \exp \left\{-\frac{1}{2}[\mathbf{r}-\hat{\mathbf{r}}]^{T} \Sigma_{\mathbf{r}}^{-1}[\mathbf{r}-\hat{\mathbf{r}}]\right\}$.

with mean $\hat{\mathbf{r}}$ and covariance matrix $\Sigma_{\mathbf{r}}$ so that the position 


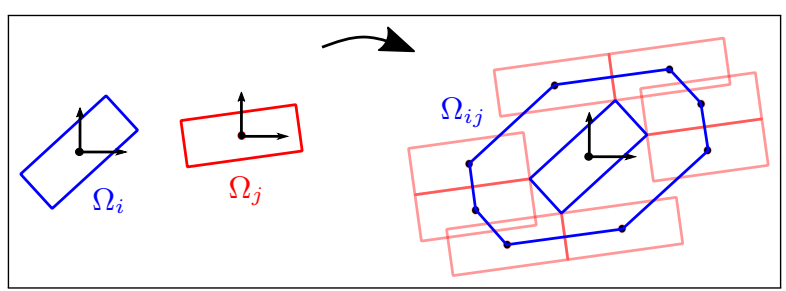

Fig. 3: Schema for determining the geometrical convolution (Minkowski difference) of two entities by placing the geometry of one entity around the corners of the other entity and taking the centers as boundary for the polygonal-shaped geometrical convolution area $\Omega_{i j}$ where the two entities would collide.

probabilities obey

$$
p_{\mathbf{r}, j}\left(\mathbf{r}_{j}\right):=\mathcal{N}\left(\mathbf{r}_{j} \mid \hat{\mathbf{r}}_{j}, \Sigma_{\mathbf{r}, j}\right) .
$$

In 2 dimensions, a collision probability between any 2 entities $i, j$ can then be calculated by moving into relative coordinates $\mathbf{d}_{i j}:=\mathbf{r}_{i}-\mathbf{r}_{j}$, e.g. in this case of the vehicle $i$. This results in a pdf for the 2-dimensional distance vector $\mathbf{d}_{i j}$.

$$
p_{\mathbf{d}, i j}\left(\mathbf{d}_{i j}\right):=\mathcal{N}\left(\mathbf{d}_{i j} \mid \hat{\mathbf{d}}_{i j}, \Sigma_{\mathbf{d}, i j}\right)
$$

with

$$
\begin{aligned}
& \hat{\mathbf{d}}_{i j}=\hat{\mathbf{r}}_{i}-\hat{\mathbf{r}}_{j} \\
& \Sigma_{\mathbf{d}, i j}=\Sigma_{\mathbf{r}, i}+\Sigma_{\mathbf{r}, j} .
\end{aligned}
$$

The distance vectors $\mathbf{d}_{i j}$, for which the two vehicles collide, can be calculated by a geometrical convolution (Minkowski difference) of the two polygons according to $\Omega_{i j}:=\Omega_{i} \times \Omega_{j}$, with boundary polyline $d \Omega_{i j}$. For this purpose, the vehicle $j$ geometry is kept fixed while the vehicle $i$ geometry is repeatedly placed around it in a way that their boundaries just touch each other. Any area covered by the position difference $\mathbf{d}_{i j}$ where $\Omega_{j}$ overlaps with $\Omega_{i}$ then results in the hull $\Omega_{i j}$. For the case of two rectangles as $\Omega_{i}$ and $\Omega_{j}$, the hull results in an octagon, and generally, it is convex if the shape polygons are also convex (see Fig. 3). All points $\mathbf{d}_{i j}$ inside of this collision area $\Omega_{i j}$ correspond to collision states, whereas all points outside are safe states. We then have that the mass within the collision area

$$
P_{\mathrm{ov}, i j}:=\iint_{\Omega_{i j}} p_{\mathbf{d}, i j}\left(\mathbf{d}_{i j}\right) d \mathbf{d}_{i j}
$$

gives us the overall probability for the 2 involved entities $i$ and $j$ to overlap, and, correspondingly, $P_{\mathrm{nonov}, i j}:=1-P_{\mathrm{ov}, i j}$ the probability that they will not overlap.

\section{B. Instantaneous and Differential Collision Probability}

We denote both $P_{\mathrm{ov}, i j}$ and $P_{\text {nonov, } i j}$ as probabilities because they are properly bounded to $[0,1]$. However, the interpretation of these quantities as "collision" probabilities is problematic: The probability calculated in such a way are valid for any time instant $t$, independently of an observation time interval length, i.e., they do not scale with time. However collision probabilities arise due to a process in time (the collision process), and should therefore depend on the observation interval $\Delta t$, as stated in (6).

This becomes clear observing that the relevant quantity for calculating the collision probability is not the total probability within the collision area (15), but instead the amount of probability that moves into the collision area over time when the scenario evolves, given by the probability density flow into the collision area, i.e., the inbound flux of $p_{\mathbf{d}, i j}\left(\mathbf{d}_{i j}\right)$ over the collision area boundary $d \Omega_{i j}$. The flux originates by both $p_{\mathbf{d}, i j}\left(\mathbf{d}_{i j}\right)$ and $\Omega_{i j}$ changing over time, the first because of e.g. relative position changes and the last because of orientation changes between the involved entities. We simplify this calculation by "truncating" the overlapping states from the pdf $p_{\mathbf{d}, i j}\left(\mathbf{d}_{i j}\right)$ at each time step, i.e., by removing those states that are inside of the collision area $\Omega_{i j}$. We then get for the instantaneous collision probability

$$
P_{\mathrm{coll}, i j}(t ; \Delta t) \approx P_{\mathrm{ov}, i j}(t)-P_{\mathrm{ov}, i j}(t-\Delta t) \approx P_{\mathrm{ov}, i j}(t) .
$$

because $P_{\mathrm{ov}, i j}(t-\Delta t)$ is zero after truncation. The truncation approach would correspond to removing those states which exhibit a physical overlap, so that these states are not considered further for the remaining prediction process. This approach is consistent with MC simulations with the probability functions approximated by a cloud of particles, removing all particles that enter the collision area at each time step.

The interesting point is that in several previous approaches [15], [16], $P_{\mathrm{ov}, i j}(t)$ was used directly (without truncation) to calculate the collision probability, which leads to effects which are inconsistent with real driving behavior, because colliding states survive for the next risk calculations. The question is now, how can we use (16) with truncation to derive fast and analytic approaches to calculate an improved estimate for collision risk probability. This approach will be described in the following sections.

\section{Truncation of 1D-Gaussian Density Functions}

Let us consider first a 1D Gaussian probability function $d \sim \mathcal{N}\left(d \mid \hat{d}, \sigma_{d}^{2}\right)$, which we truncate at either a lower or an upper bound $b^{-}, b^{+}$or both, meaning that we set all values to zero outside of $b^{-} \leq x \leq b^{+}$. The question is: What would a good Gaussian approximation of this truncated probability distribution? That is, we want to know the variance and mean of the Gaussian function that we can use to approximate the truncated Gaussian. This is a well-known problem from standard statistical literature [19], leading to

$$
\begin{aligned}
\hat{d}_{\text {trunc }} & =\hat{d}-\sigma_{d}\left[\frac{\phi\left(b^{+}\right)-\phi\left(b^{-}\right)}{\Phi\left(b^{+}\right)-\Phi\left(b^{-}\right)}\right] \\
\sigma_{d, \text { trunc }}^{2} & =\sigma_{d}^{2}\left[1-\frac{\alpha^{+} \phi\left(b^{+}\right)-\alpha^{-} \phi\left(b^{-}\right)}{\Phi\left(b^{+}\right)-\Phi\left(b^{-}\right)}\right]-\left(\hat{d}_{\text {trunc }}-\hat{d}\right)^{2}
\end{aligned}
$$

where we use

$$
\begin{aligned}
& \alpha^{ \pm}:=\frac{b^{ \pm}-\hat{d}}{\sigma_{d}} \\
& \phi(x):=\frac{1}{\sqrt{2 \pi}} e^{-\frac{1}{2} x^{2}} \quad \Phi(x):=\int_{0}^{x} \phi\left(x^{\prime}\right) d x^{\prime}
\end{aligned}
$$


with the standard normal distribution $\phi$ and the associated standard cumulative distribution $\Phi$.

If $b^{ \pm}$indicates the boundaries of a forbidden region $\Omega$ like e.g. the previously introduced collision area, with $d \in \Omega$ meaning collision, we additionally get that

$$
P_{\mathrm{ov}}=\left[\Phi\left(\alpha^{+}\right)-\Phi\left(\alpha^{-}\right)\right]
$$

is the probability that e.g. there is a geometric overlap between the 2 entities involved in a potential collision.

The one-sided truncation with $b^{ \pm}=\mp \infty$ can then be extracted as a special case.

\section{Calculation of Collision Area Probability Distribution}

With the truncation method described above, we are able to get a representation of the collision area pdf, which we will later use for obtaining the non-collision area representation and for calculating the collision probability at the current predicted time point. For convex entity polygons, the collision area $\Omega_{i j}$ gained by geometrical convolution described in section IV-A, is also a convex polygon. For that polygon, we can use the special case of one-sided truncation for each polygon edge. If we assume rectangular vehicle shapes, the geometrical convolution is a point symmetric polygon with pairs of parallel edges, so that we can use the two-sided truncation method. We truncate the pdf in relative coordinate space $p_{\mathbf{d}, i j}\left(\mathbf{d}_{i j}\right)$ (14), where the edges $\mathbf{a}^{T} \mathbf{d}=b$ of the geometrical convolution are represented, by projecting them into the $1 \mathrm{D}$ case $\mathcal{N}\left(d \mid \hat{d}, \sigma_{d}^{2}\right)$, so that the truncation methods from the previous section can be applied to remove all parts outside the collision area. The transformation equations are as follows:

$$
\begin{aligned}
\hat{d} & =\mathbf{a}^{T} \hat{\mathbf{d}} \\
\sigma_{d}^{2} & =\mathbf{a}^{T} \Sigma_{\mathbf{d}} \mathbf{a}
\end{aligned}
$$

With (20) we can apply (17) to get the truncated mean $\hat{d}_{\text {trunc }}$ and variance $\sigma_{d \text {,trunc }}$ in $1 \mathrm{D}$. The mean $\hat{\mathbf{d}}_{\text {trunc }}$ and variance $\Sigma_{\mathbf{d} \text {,trunc }}$ of the multivariate Gaussian in $2 \mathrm{D}$ space $\mathcal{N}\left(\mathbf{d} \mid \hat{\mathbf{d}}_{\text {trunc }}, \Sigma_{\mathbf{d} \text {,trunc }}\right)$ can be calculated as follows [18]:

$$
\begin{aligned}
\hat{\mathbf{d}}_{\text {trunc }} & :=\hat{\mathbf{d}}-\frac{\Sigma_{\mathbf{d}} \mathbf{a}}{\sigma_{d}^{2}}\left(\mathbf{a}^{T} \hat{\mathbf{d}}-\hat{d}_{\text {trunc }}\right) \\
\Sigma_{\mathbf{d}, \text { trunc }} & :=\Sigma_{\mathbf{d}}-\frac{\Sigma_{\mathbf{d}} \mathbf{a}}{\sigma_{d}^{2}}\left(\sigma_{d}^{2}-\sigma_{d, \text { trunc }}^{2}\right) \frac{\mathbf{a}^{T} \Sigma_{\mathbf{d}}}{\sigma_{d}^{2}}
\end{aligned}
$$

For the composition of several linear constraints, we calculate the corresponding $P_{\mathrm{ov}}$ 's from the original nontruncated distribution $p_{\mathbf{d}, i j}\left(\mathbf{d}_{i j}\right)$ for all constraints, beginning in an ordered way by truncating the distribution with the highest $P_{\mathrm{ov}}$. The truncated pdf is then used as input pdf $p_{\mathbf{d}, i j}\left(\mathbf{d}_{i j}\right)$ for the next truncation. To calculate the $P_{\mathrm{ov}, i j}$ for (15), we multiply all overlap collision probabilities $P_{\mathrm{ov}}$ (eq. (19)) gained from the different truncation processes, where we use both finite borders $\alpha^{+}$and $\alpha^{-}$for parallel collision area edges.

\section{E. Calculation of Non-Collision Probability Distribution}

We have seen in the last section how to calculate the collision area probability, representing the states that will be involved in the collision. What we are interested in, however, is the complement, i.e. the probability $p_{\mathbf{d}, \mathrm{nc}}(\mathbf{d})$ that we will not be engaged in a collision. This is given by removing all collision area states from the state pdf. Since this cannot be done directly in an analytic way (for a comparison with MC simulations see the evaluation section V), we use the truncation approximation to achieve this. We therefore take the truncated pdf calculated as detailed in section IV as approximation for the collision area pdf. We also calculate the total probability $P_{\text {coll }, i j}$ from the truncation as explained in the previous section and use eq. (16) to get the collision probability $P_{\text {coll }}$. We then set

$$
p_{\mathbf{d}}(\mathbf{d}) \approx P_{\text {coll }} p_{\mathbf{d}, \text { trunc }}(\mathbf{d})+\left(1-P_{\text {coll }}\right) p_{\mathbf{d}, \mathrm{nc}}(\mathbf{d})
$$

i.e., we approximate the total Gaussian pdf as a sum of the truncation approximation of the collision area pdf and the rest, representing the non-collided states. The mean and the variance of all Gaussian pdf's are gained by the conservation of statistical moments in the following way:

$$
\begin{aligned}
\hat{\mathbf{d}} & =\int p_{\mathbf{d}}(\mathbf{d}) \mathbf{d} d \mathbf{d} \\
& =P_{\text {coll }} \hat{\mathbf{d}}_{\text {trunc }}+\left(1-P_{\text {coll }}\right) \hat{\mathbf{d}}_{\mathrm{nc}} \\
\Sigma_{\mathbf{d}} & =\int p_{\mathbf{d}}(\mathbf{d})(\mathbf{d}-\hat{\mathbf{d}})(\mathbf{d}-\hat{\mathbf{d}})^{T} d \mathbf{d} \\
& =P_{\text {coll }}\left[\Sigma_{\mathbf{d}, \text { trunc }}+\left(\hat{\mathbf{d}}_{\text {trunc }}-\hat{\mathbf{d}}\right)\left(\hat{\mathbf{d}}_{\text {trunc }}-\hat{\mathbf{d}}\right)^{T}\right] \\
+ & \left(1-P_{\text {coll }}\right)\left[\Sigma_{\mathbf{d}, \mathrm{nc}}+\left(\hat{\mathbf{d}}_{\mathrm{nc}}-\hat{\mathbf{d}}\right)\left(\hat{\mathbf{d}}_{\mathrm{nc}}-\hat{\mathbf{d}}\right)^{T}\right]
\end{aligned}
$$

These equations can finally be solved for $\hat{\mathbf{d}}_{\mathrm{nc}}$ and $\Sigma_{\mathbf{d}, \mathrm{nc}}$, which are the parameters of our pdf $p_{\mathbf{d}, \text { nc }}(\mathbf{d})$ for non-collided states, approximated by a Gaussian. We then get:

$$
\begin{aligned}
\hat{\mathbf{d}}_{\mathrm{nc}}= & \frac{1}{1-P_{\text {coll }}}\left(\hat{\mathbf{d}}-P_{\text {coll }} \hat{\mathbf{d}}_{\text {trunc }}\right) \\
\Sigma_{\mathbf{d}, \mathrm{nc}}= & -\frac{P_{\text {coll }}}{1-P_{\text {coll }}}\left[\Sigma_{\mathbf{d}, \text { trunc }}+\left(\hat{\mathbf{d}}_{\text {trunc }}-\hat{\mathbf{d}}\right)\left(\hat{\mathbf{d}}_{\text {trunc }}-\hat{\mathbf{d}}\right)^{T}\right] \\
& +\frac{1}{1-P_{\text {coll }}} \Sigma_{\mathbf{d}}-\left(\hat{\mathbf{d}}_{\mathrm{nc}}-\hat{\mathbf{d}}\right)\left(\hat{\mathbf{d}}_{\mathrm{nc}}-\hat{\mathbf{d}}\right)^{T}
\end{aligned}
$$

These parameters determine the Gaussian which can be transferred into the next predicted time step by a given transition model.

\section{F. Single Entity Probability Truncated Distribution}

In the previous section, we have seen how to calculate the distribution in differential state space and how to determine and remove the states that are prone to collision. This was done based on a Gaussian approximation and a truncation approach, which allows us to estimate this in a computationally efficient way.

Once we have calculated the parameters $\hat{\mathbf{d}}_{\mathrm{nc}}$ and $\Sigma_{\mathbf{d}, \mathrm{nc}}$ of the reduced pdf, we have to estimate the state pdf's of the single entities. In case that one of them is deterministic or has 
a substantially smaller variance than the other, e.g. $\Sigma_{\mathbf{r}, i} \ll$ $\Sigma_{\mathbf{r}, j}$, it is approximately $\Sigma_{\mathbf{d}} \approx \Sigma_{\mathbf{r}, j}$ and we can directly take

$$
\begin{aligned}
\hat{\mathbf{r}}_{\mathrm{nc}, j} & \approx \mathbf{r}_{i}+\hat{\mathbf{d}}_{\mathrm{nc}} \\
\Sigma_{\mathbf{r}, \mathrm{nc}, j} & \approx \Sigma_{\mathrm{d}, \mathrm{nc}} .
\end{aligned}
$$

In case that both $\Sigma_{\mathbf{r}, j}$ and $\Sigma_{\mathbf{r}, i}$ are probabilistic and have to be correctly considered, the idea is similar but we have to proceed in a mathematically more general way. In section IV-C, we did the projection to the $1 \mathrm{D}$ distance by 2 steps,

$$
\left[\mathbf{r}_{i}, \mathbf{r}_{j}\right] \rightarrow \mathbf{d} \rightarrow d,
$$

with the help of the 2D relative position vector $\mathbf{d}=\mathbf{r}_{i}-\mathbf{r}_{j}$ and the projection $\mathbf{a}^{T} \mathbf{d}$. Once we are in the $\mathbf{d}$ space, the information regarding any single traffic participant is lost, so that after a truncation the correct assignment of truncation components to each contribution $i$ and $j$ cannot be retrieved anymore.

Therefore, instead of using $\mathbf{d}$, we stay in the combined state space (shown here for $i$ and $j$ positions, but generalizable to arbitrary number of traffic participants and state variables)

$$
\mathbf{x}^{T}=\left[\mathbf{r}_{i}^{T}, \mathbf{r}_{j}^{T}\right],
$$

with $\mathbf{x} \sim \mathcal{N}\left(\mathbf{x} \mid \hat{\mathbf{x}}, \Sigma_{\mathbf{x}}\right)$ and mean and variance

$$
\begin{aligned}
\hat{\mathbf{x}}^{T} & =\left[\hat{\mathbf{r}}_{i}^{T}, \hat{\mathbf{r}}_{j}^{T}\right] \\
\Sigma_{\mathbf{x}} & =\operatorname{boxdiag}\left(\Sigma_{\mathbf{x}, i}, \Sigma_{\mathbf{x}, j}\right) .
\end{aligned}
$$

Analogously as before in section IV, we impose e.g. linear (hyper-plane) constraints there by $A^{T} \mathbf{x} \leq b$. We then project again into the 1D Gaussian distribution $\mathcal{N}\left(d \mid \hat{d}, \sigma_{d}^{2}\right)$ by $d:=$ $A^{T} \mathrm{x}$ so that

$$
\begin{aligned}
\hat{d} & =A^{T} \hat{\mathbf{x}} \\
\sigma_{d}^{2} & =A^{T} \Sigma_{\mathbf{x}} A
\end{aligned}
$$

This results in new mean (like in eq. (21))

$$
\hat{\mathbf{d}}_{\text {trunc }}:=\hat{\mathbf{x}}-\frac{\Sigma_{\mathbf{x}} A}{\sigma_{d}^{2}}\left(A^{T} \hat{\mathbf{x}}-\hat{d}_{\text {trunc }}\right)
$$

and variance (like in (22))

$$
\Sigma_{\mathbf{d}, \text { trunc }}:=\Sigma_{\mathbf{x}}-\frac{\Sigma_{\mathbf{x}} A}{\sigma_{d}^{2}}\left(\sigma_{d}^{2}-\sigma_{d, \text { trunc }}^{2}\right) \frac{A^{T} \Sigma_{\mathbf{x}}}{\sigma_{d}^{2}} .
$$

For the constraint parameter $A$, we remark that the constraint in joint state space, $A^{T} \mathbf{x}=b$, can be expressed as

$$
A^{T} \mathbf{x}=\mathbf{a}^{T} T \mathbf{x}=\mathbf{a}^{T} \mathbf{d}
$$

with the matrix $T$ which transforms from joint state space $\mathbf{x}$ to the $j$-relative state space $\mathbf{d}$ via $\mathbf{d}:=T \mathbf{x}$. From this, we can directly calculate the joint space constraint vector from the $2 \mathrm{D}$ parameter $\mathrm{a}$ by

$$
A=T^{T} \mathbf{a}
$$

and calculate the joint state truncated pdf according to eqs. (33) and (34). This results in the parameters of the truncated Gaussian distribution as desired.
To finalize, from the joint state pdf we have to calculate again the single entity truncated position pdf's. This can be done in a straightforward way by marginalizing over the variables that are not of interest. E.g. if we want to extract the position $\mathbf{r}_{j}$ pdf, we have to marginalize $\mathcal{N}\left(\mathbf{x} \mid \hat{\mathbf{x}}, \Sigma_{\mathbf{x}}\right)$ over $\mathbf{r}_{i}$. This results again in a Gaussian distribution, and its mean and variance can be calculated according to [20], where we take the components of the gained overall non collided distribution representation corresponding to their vehicle's identity. The vehicle's mean vector $\hat{\mathbf{r}}_{\mathrm{nc}, j}$ is placed on the $\mathrm{j}$ 'th position of the overall mean vector $\hat{\mathbf{x}}_{\mathrm{nc}}$ and the vehicle's variance $\Sigma_{\mathbf{r}, \mathrm{nc}, j}$ is placed on the $\mathrm{j}$ 'th positon of the overall variance $\Sigma_{\mathbf{x}, \text { nc }}$ main diagonal (compare eq. (31)).

\section{G. N-Entity Instantaneous Collision Probability}

For any 2 entities and at any time step, the full procedure to apply the pdf truncation in one time step then is as follows (compare to Fig. 2):

1) Start with entity state pdf's $p_{\mathbf{r}, i}\left(\mathbf{r}_{i}\right)$ and oriented shape representation $\Omega_{j}$.

2) Transformation into pairwise relative space $\mathbf{d}_{i j}$ and determination of collision area $\Omega_{i j}$ with entity shapes (shown in section IV-A) to obtain linear representation of edges.

3) Transformation of overall state pdf $p(\mathbf{x})$ into 1D (32) with (36) and truncation by linear (parallel) constraints afterwards with (17).

4) Iterative determination of means (33) and variances (34) of the overall state pdf $p_{\mathbf{x} \text {,trunc }}(\mathbf{x})$ for each edge (pair).

5) Calculation of mean (26) and variance (27) while preserving statistical moments to obtain the non-collided state pdf representation.

6) Iterative repetition of step 1) to 5) for all entity pairs.

7) Marginalization to obtain the entity state pdf's for transition execution.

The collision probability magnitude and the integral risk measure is finally gained by calculating successively:

1) Overlap probability $P_{\mathrm{ov}, i j}$ with (15)

2) Collision probability $P_{\text {coll }, i j}$ with (16)

3) Survival probability $P_{\text {surv }, i}$ with (2) \& (5)

4) Time-course sensitive prob.'s $P_{\text {tcscoll }, i j}$ with (9) and

5) Integrated probability measure $P_{\text {intcoll }, i j}$ with (10).

In a setting with several other entities, only collisions with the ego vehicle leads to a truncation of the overall pdf. Mutual collisions among the others are neglected and do not lead to any adaptation of the overall pdf.

\section{Evaluation}

In the previous sections, we have seen how the Survival Theory can be extended to adapt to the shape deformation of the predicted state probability distributions by truncation and Gaussian approximation with conservation of moments (COM). In this section, we show the applicability of our approach to driving scenarios. For this purpose, we compare the proposed survival approach ("truncated survival") with 


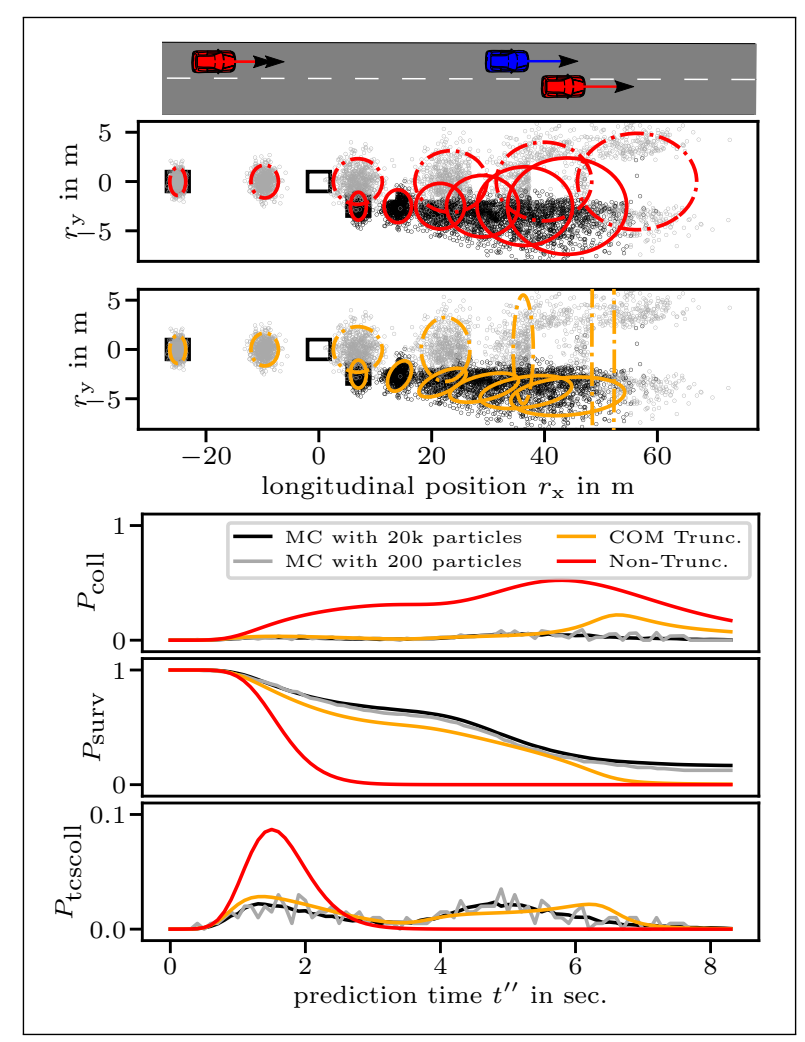

Fig. 4: Three-vehicle tailgating and overtaking scenario. Top: scenario, where the ego vehicle (blue) is overtaking another vehicle on a neighbouring lane and a second vehicle is arriving with high velocity on the ego-lane. 2nd row: Predicted Gaussian $2 \sigma-$ ellipsoid potentials for the survival approach without truncation (red) and MC particles (black and grey). 3rd row: The survival approach with truncation (orange) and MC particles. The Gaussians from the truncation approach provide a good approximation for MC particles. 4th-6th row: Instantaneous, survival and time-course sensitive collision probability for different probability models. One can see that the collision probability of the truncation model is very similar to the MC results in overtaking scenarios, but deviates when multi-modal distributions develop.

the plain survival approach without shape adaptation ("nontruncated survival") and MC simulations.

First, we investigate the approximation of the pdf's by showing the shape and instantaneous collision probability over prediction time in high risk scenarios, shown in Fig. 4 (top), containing two scenarios: An ego vehicle (blue) is passing another vehicle and is subsequently tailgated by a third vehicle. In Fig. 4 (bottom), we show the ego-vehicle's instantaneous, survival and time-course sensitive probability curves, and it can be seen that the truncated survival approach (orange line) matches the MC simulations better (black) than the non-truncated survival approach. However, at prediction times $s>6 \mathrm{sec}$, there is a deviation caused by the splitting of the grey MC particles into 2 streams (Fig. 4, third row), which our unimodal Gaussian cannot handle appropriately. It can also be seen from the survival probabilities' faster decay to zero, that the non-truncated survival approach largely overestimates the collision risk during the tailgating process. On an i7-2600 processor with 4 cores our algorithm is around

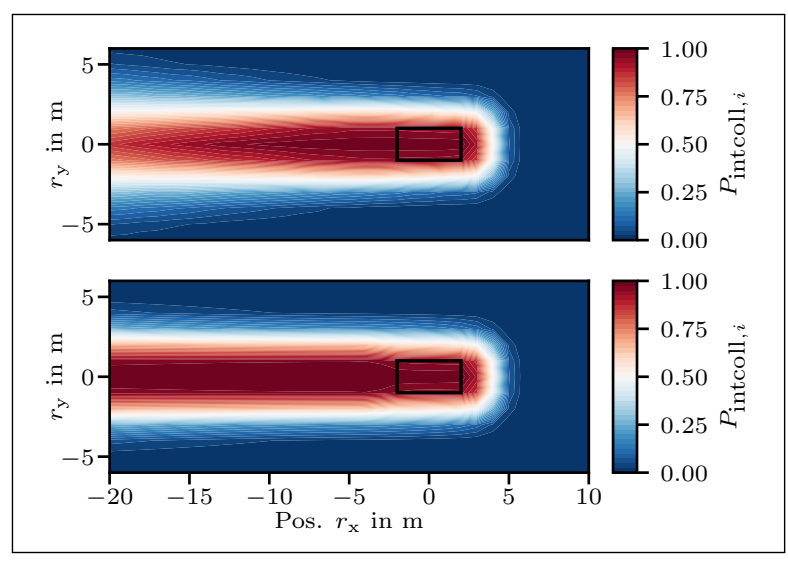

Fig. 5: Two-vehicle driving scenario, where the ego vehicle starting point is at different relative positions to another vehicle (black rectangle). The ego vehicle is driving faster with a relative horizontal velocity of $10 \mathrm{~m} / \mathrm{s}$. At every point, the integral collision probability $P_{\text {intcoll }, i}(t)$ is calculated to form a spatial heat map. Top: MC simulation. Bottom: Truncated survival model according to eq. (10).

100 times faster than a MC simulation with 20.000 particles (similar computational time with around 200 particles).

In a second evaluation, we investigated whether the overall risk as estimated by the truncation approach matches that of the MC simulations. For this purpose, we used the integral collision probability eq. (10), which is basically the expectation value that a collision will occur over the entire predicted future. This is done for a scenario where the egovehicle is approaching another vehicle from left to right, starting at different spatial points with a constant relative velocity. For each spatial point, we then get a scalar quantity $P_{\text {intcoll }, i}(t)<1$ indicating the overall integral collision probability, resulting in a sort of spatial heat map. Fig. 5 shows results from the MC simulation (top) vs. the truncation approach (bottom). We again see the good match, with some overestimation of the overall risk by our truncation approach.

To investigate overtaking tendencies for fixed parallel offsets, we calculate the velocity-dependent integral collision measurement (see Fig. 6). Our approach matches generally well with some deviations in case of small lateral distances and small relative velocities, where it is overestimating the integral collision measurement $P_{\text {intcoll }, i}$ in these special cases.

The last scenario shows the positional heatmap of our integral collision measurement $P_{\text {intcoll, } i}$ in a left to right driving ego vehicle setting with two other, differently shaped vehicles (see Fig. 7). One can see the overestimation of our model (bottom) for high longitudinal distances but a similar heatmap in the critical valley passage, in the middle of the two blocking vehicles, compared to MC simulations (top).

\section{SUMMARY AND CONCLUSIONS}

We have presented a model for the analytic shape estimation of the future state probability distribution functions with the purpose of improving predictive collision risk estimation for behavior planning. We introduced a truncation approximation which allows to work efficiently on the basis 


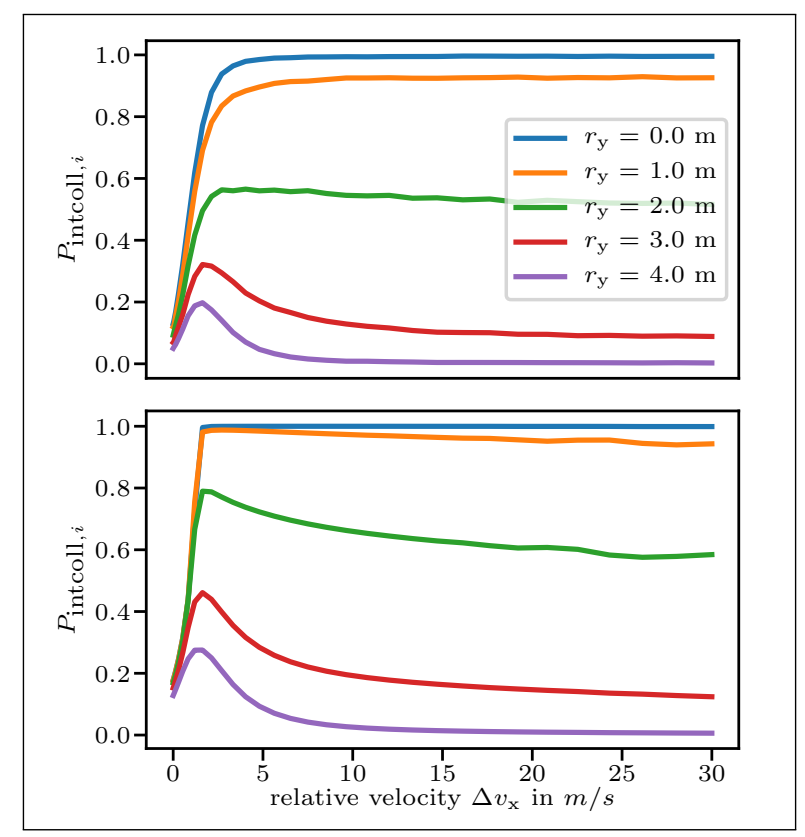

Fig. 6: Integral collision probability measurements $P_{\text {intcoll }, i}$ in a two-vehicle following scenario, where the ego vehicle starts at different relative lateral distances $r_{y}$ and relative velocities $\Delta v_{x}$ to the frontal vehicle at a fixed longitudinal relative initial distance $\Delta x=10 \mathrm{~m}$. Top: Integral probability values calculated by MC simulation. Bottom: Truncated survival approach. For larger distances and velocity differences the match is very good, however, for small spatial distances and small velocities, the unimodality of the Gaussian leads to deviations.

of Gaussian state pdf's. The approach is consistent with and improves a previously presented general risk estimation approach based on survival probability theory. In simulations of different scenarios, including multi-vehicle risks as well as longitudinal and lateral collisions, we have shown that our introduced integral collision probability measurement considers the entire predicted trajectory and leads to reasonable heatmaps. Additionally, our approach matches well to the results of MC simulations in various scenarios, as long as the resulting true distributions can remain unimodal. Our approach is computationally fast, scales linearly by the amount of involved vehicles and creates non-zero risk also in far-distant scenarios, where particle density is low. In deviating cases, even if it overestimates the overall risk, the presented approach is always better than approaches which do not consider a shape adaptation of the state pdf's.

\section{REFERENCES}

[1] S. Luko, "Risk management principles and guidelines," Quality Engineering, vol. 25, Oct 2013.

[2] J. S. Park, C. Park, and D. Manocha, "Efficient probabilistic collision detection for non-convex shapes," in 2017 IEEE International Conference on Robotics and Automation (ICRA). IEEE, 2017, pp. 1944-1951.

[3] M. Prandini, J. Hu, J. Lygeros, and S. Sastry, "A probabilistic approach to aircraft conflict detection," IEEE Transactions on Intelligent Transportation Systems, vol. 1, no. 4, pp. 199-220, Dec 2000.

[4] J. Park and J. Kim, "Predictive evaluation of ship collision risk using the concept of probability flow," IEEE Journal of Oceanic Engineering, vol. 42, no. 4, pp. 836-845, 2016.

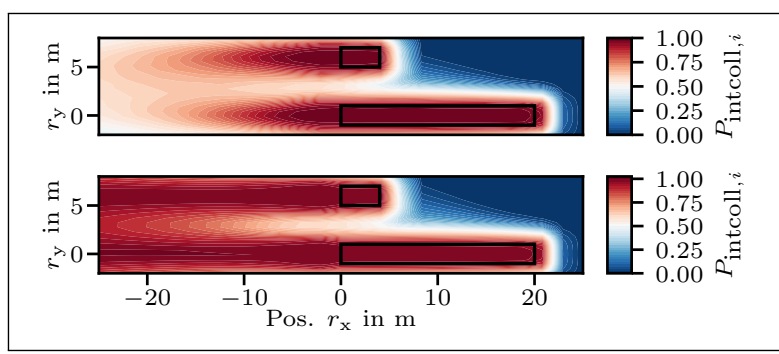

Fig. 7: Integral collision probability $P_{\text {intcoll }, i}$ heat map in a tailgating scenario, where the ego vehicle approaches two other blocking vehicles from the left. Top: Integral probability values calculated by a MC Simulation. Bottom: Truncated survival approach, showing a similar heat map appearance while overestimating the risk between the two blocking vehicles.

[5] C. Hubmann, M. Becker, D. Althoff, D. Lenz, and C. Stiller, "Decision making for autonomous driving considering interaction and uncertain prediction of surrounding vehicles," in 2017 IEEE Intelligent Vehicles Symposium, 2017, pp. 1671-1678.

[6] M. Schreier, "Bayesian environment representation, prediction, and criticality assessment for driver assistance systems," atAutomatisierungstechnik, vol. 65, no. 2, pp. 151-152, 2017.

[7] B. Ristic, S. Arulampalam, and N. Gordon, "Beyond the kalman filter," IEEE Aerospace and Electronic Systems Magazine, vol. 19, no. 7, pp. 37-38, 2004.

[8] C. Park, J. S. Park, and D. Manocha, "Fast and bounded probabilistic collision detection for high-dof trajectory planning in dynamic environments," IEEE Transactions on Automation Science and Engineering, vol. 15, no. 3, pp. 980-991, 2018.

[9] N. E. Du Toit and J. W. Burdick, "Probabilistic collision checking with chance constraints," IEEE Transactions on Robotics, vol. 27, no. 4, pp. 809-815, 2011.

[10] A. Lambert, D. Gruyer, and G. Saint Pierre, "A fast monte carlo algorithm for collision probability estimation," in 2008 10th International Conference on Control, Automation, Robotics and Vision, Dec 2008, pp. $406-411$.

[11] J. Eggert, S. Klingelschmitt, and F. Damerow, "The foresighted driver: Future adas based on generalized predictive risk estimation," in FASTzero 2015 Symposium, B. K. Jonas Fredriksson and J. Sjöberg, Eds. Chalmers University Technology, Gothenburg, Sweden, September 2015, pp. 93-100.

[12] D. Althoff, "Safety assessment for motion planning in uncertain and dynamic environments," Ph.D. dissertation, Technische Universität München, 2014.

[13] R. Altendorfer and C. Wilkmann, "A new approach to estimate the collision probability for automotive applications," arXiv: Systems and Control, 2019.

[14] J. Eggert, "Predictive risk estimation for intelligent adas functions," in 17th International IEEE Conference on Intelligent Transportation Systems, 2014, pp. 711-718.

[15] J. Eggert and F. Müller, "A foresighted driver model derived from integral expected risk," in 2019 IEEE Intelligent Transportation Systems Conference, 2019, pp. 1223-1230.

[16] F. Müller and J. Eggert, "Behaviour investigation of a risk-aware driving model for trajectory prediction," in FAST-zero 2019 Symposium, September 2019.

[17] C. Rodemerk, S. Habenicht, A. Weitzel, H. Winner, and T. Schmitt, "Development of a general criticality criterion for the risk estimation of driving situations and its application to a maneuver-based lane change assistance system," in 2012 IEEE Intelligent Vehicles Symposium. IEEE, 2012, pp. 264-269.

[18] S. Patil, J. Van Den Berg, and R. Alterovitz, "Estimating probability of collision for safe motion planning under gaussian motion and sensing uncertainty," in 2012 IEEE International Conference on Robotics and Automation. IEEE, 2012, pp. 3238-3244.

[19] N. L. Johnson, S. Kotz, and N. Balakrishnan, Continuous univariate distributions. John Wiley \& Sons, Ltd, 1994.

[20] C. M. Bishop, Pattern recognition and machine learning. springer, 2006, ch. 2, pp. 88-90. 